\title{
A new prototype melt-electrospinning device for the production of biobased thermoplastic sub-microfibers and nanofibers
}

\author{
Kylie Koenig ${ }^{*}$ (D) Konrad Beukenberg, Fabian Langensiepen and Gunnar Seide
}

\begin{abstract}
Sub-microfibers and nanofibers have a high surface-to-volume ratio, which makes them suitable for diverse applications including environmental remediation and filtration, energy production and storage, electronic and optical sensors, tissue engineering, and drug delivery. However, the use of such materials is limited by the low throughput of established manufacturing technologies. This short report provides an overview of current production methods for sub-microfibers and nanofibers and then introduces a new melt-electrospinning prototype based on a spinneret with 600 nozzles, thereby providing an important step towards larger-scale production. The prototype features an innovative collector that achieves the optimal spreading of the fiber due to its uneven surface, as well as a polymer inlet that ensures even polymer distribution to all nozzles. We prepared a first generation of biobased fibers with diameters ranging from 1.000 to $7.000 \mu \mathrm{m}$ using polylactic acid and $6 \%(w / w)$ sodium stearate, but finer fibers could be produced in the future by optimizing the prototype and the composition of the raw materials. Melt electrospinning using the new prototype is a promising method for the production of high-quality sub-microfibers and nanofibers.
\end{abstract}

Keywords: Fiber spinning, Nanotechnology, Polylactic acid, Nanofiber nonwoven, Eco-friendly production, Melt spinning, Fiber production, Electrospinning, Process development

\section{Introduction}

Nanotechnology can be generally defined as the development, handling and control of structures or materials with at least one dimension within the size range $1-100 \mathrm{~nm}$, and the advent of precision tools for nanoscale engineering has promoted great interest in this emerging field over the last 30 years $[1,2]$. Nanotechnology exploits the properties of materials that depend on size or structure, particularly properties that differ from the behavior of individual atoms/molecules or larger masses of the same material [2]. The term "nanofiber" is frequently used in the literature to describe very thin fibers without a specified size limit, but a stricter definition as used by the Deutsches Institut für Normung (DIN) standard among

\footnotetext{
* Correspondence: kylie.konig@maastrichtuniversity.nl

Aachen-Maastricht Institute for Biobased Materials (AMIBM), Maastricht University, Brightlands Chemelot Campus, Urmonderbaan 22, 6167, RD, Geleen, The Netherlands
}

(c) The Author(s). 2019 Open Access This article is distributed under the terms of the Creative Commons Attribution 4.0 International License (http://creativecommons.org/licenses/by/4.0/), which permits unrestricted use, distribution, and reproduction in any medium, provided you give appropriate credit to the original author(s) and the source, provide a link to the Creative Commons license, and indicate if changes were made. The Creative Commons Public Domain Dedication waiver (http://creativecommons.org/publicdomain/zero/1.0/) applies to the data made available in this article, unless otherwise stated. sions and a third external dimension that is considerably larger than the nanoscale [3]. However, a comparison of many studies shows that the same term is often used as soon as the fiber diameter falls below $1 \mu \mathrm{m}$ [4]. Although such fibers are not nanofibers according to the DIN standard, the designation has become established and consolidated in recent years. With respect to the DIN standard, another term used to describe fibers with a diameter in the hundreds of nanometers range is "sub-microfiber".

The small diameter of sub-microfibers and nanofibers provides a high surface-to-volume ratio while maintaining or even improving flexibility compared to conventional fibers. Additionally, many production methods yield porous fibers thus increasing the surface area even further [5]. These properties make such fibers extremely versatile. Their diverse applications include air and water filtration $[6,7]$, the separation of water/oil and air/oil 

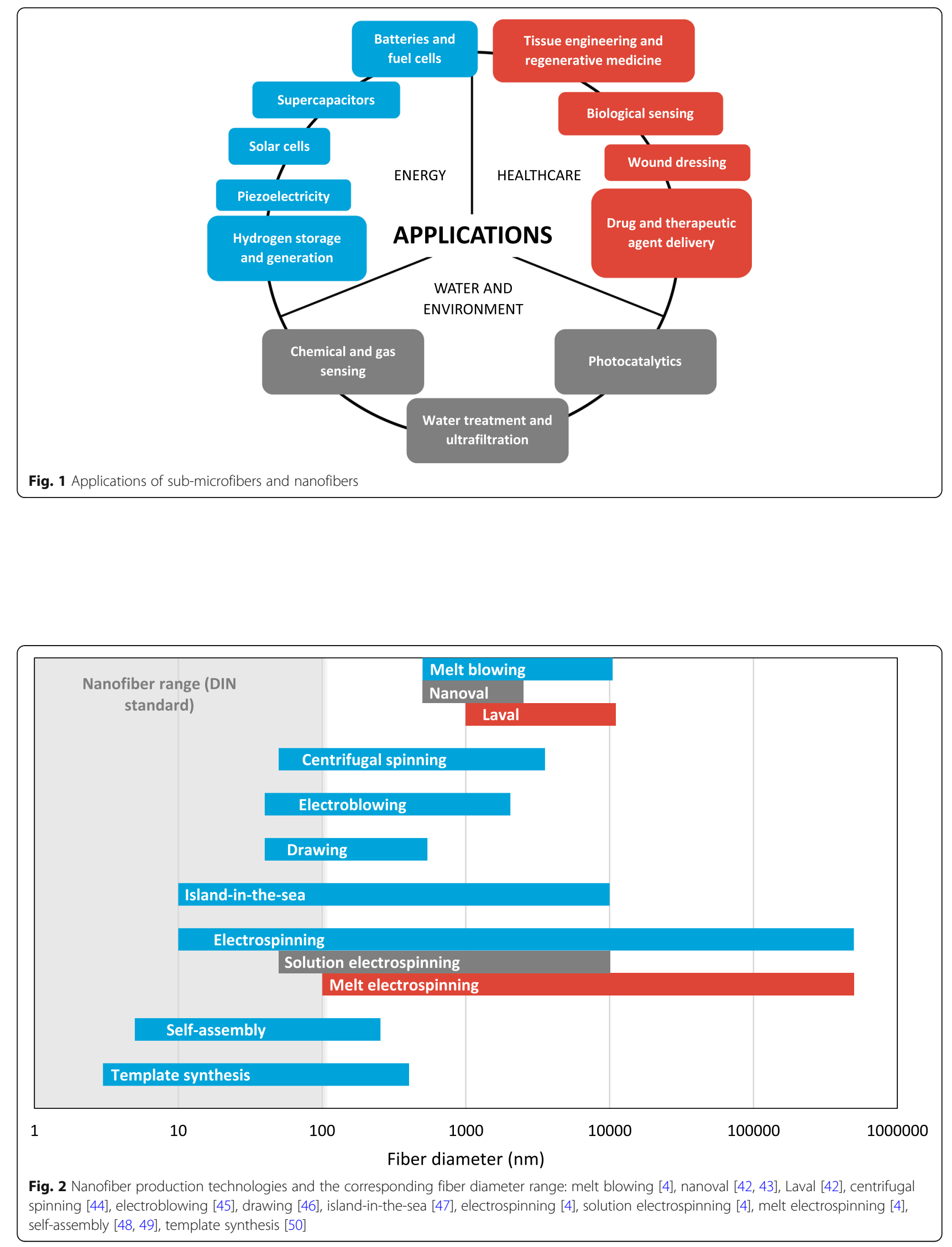
mixtures $[4,8]$, technical uses such as the development of lithium-air batteries [9-12], optical sensors [13] and textiles [5, 14], and medical applications such as tissue engineering [15-23], drug delivery [18, 19, 24-36], and the diagnosis and treatment of cancer [27, 36-40] (Fig. 1) [41]. The applications of sub-microfibers and nanofibers depend on their physical and mechanical properties, which in turn depend on the manufacturing process. This short report provides an overview of current production methods before describing a novel and scalable melt-electrospinning prototype device and its deployment for the processing of biobased materials into fibers.

\section{Current manufacturing processes for sub- microfibers and nanofibers}

Sub-microfibers and nanofibers can be produced from a range of biomaterials, such as polysaccharides (e.g. chitosan, cellulose, or alginate) and proteins (e.g. gelatin, keratin, or collagen), as well as synthetic polymers, such as polycaprolactone (PCL), polyurethane (PU), polylactic acid (PLA), and poly(lactic-co-glycolic) acid (PLGA). Figure 2 provides an overview of current major nanofiber production technologies and the fiber diameters that have typically been achieved using those methods. Only the most common processes are mentioned and there are many variants of these methods that we do not discuss in detail [42-50].

Electrospinning is the most common production method for sub-microfibers and nanofibers, and two fundamental techniques can be distinguished: solution electrospinning and melt electrospinning. Electrospinning combines a strong electrostatic field with the principle of Taylor cone formation. When a droplet of a liquid becomes charged in a field of sufficient strength, the electrostatic repulsion is strong enough to overcome the surface tension and the droplet is stretched. If the charge reaches a certain threshold, a

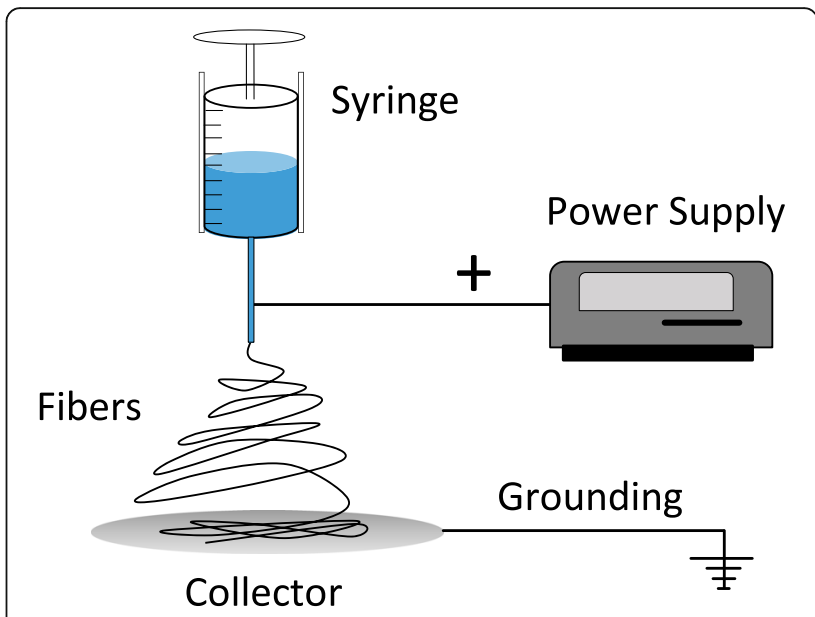

Fig. 3 Basic setup of the electrospinning production method

jet erupts from the liquid droplet and this is known as a Taylor cone [51-53]. If the liquid is viscous and cohesive, the jet does not break up into droplets (the principle of electro-spraying) but forms an electrically charged laminar jet, which elongates due to electrostatic repulsion. The jet dries (in the case of solution electrospinning) or cools sufficiently to become solid (in the case of melt electrospinning) and a nanoscale fiber is produced [54]. The basic setup for electrospinning is shown in Fig. 3. Solution electrospinning is used more frequently than melt electrospinning for the production of nanofibers because a smaller fiber diameter can be achieved (high hundreds of nanometers), and the equipment has a simpler design and higher productivity compared to current melt electrospinning devices [55]. The finest fiber produced by melt electrospinning thus far was $80 \mathrm{~nm}$ in diameter [56], although this in not yet routine and typically the fiber diameter is $>2 \mu \mathrm{m}$ [45]. The major advantage of melt electrospinning is that it does not require a solvent, avoiding any risk of toxic solvents being carried

Table 1 Overview of electrospinning methods, typical fiber diameters and applications

\begin{tabular}{|c|c|c|c|c|}
\hline Publication & $\begin{array}{l}\text { Production } \\
\text { method }\end{array}$ & Polymer & $\begin{array}{l}\text { Fiber diameter } \\
{[\mathrm{nm}]}\end{array}$ & Application \\
\hline $\begin{array}{l}\text { Wang } 2002 \\
\text { [12] }\end{array}$ & Solution & $\begin{array}{l}\text { Poly(acrylic acid) - poly } \\
\text { (pyrene methanol) }\end{array}$ & $100-300$ & Optical sensors \\
\hline Li 2012 [87] & Melt (laser melt) & Poly(L-lactic acid) & $2000-7000$ & Biomedical \\
\hline $\begin{array}{l}\text { Dalton } 2006 \\
\text { [89] }\end{array}$ & Melt & $\begin{array}{l}\text { Poly(ethylene glycol) and } \\
\text { poly(epsilon-caprolactone) }\end{array}$ & & \\
\hline Yoon 2013 [90] & Melt and S/M-hybrid & $\begin{array}{l}\text { Silk fibroin and poly } \\
\text { (L-lactic acid) }\end{array}$ & $\begin{array}{l}\text { PLA: } 8900 \\
\text { SF: } 820\end{array}$ & Biomedical (scaffolding) \\
\hline Zhou 2006 [91] & Melt & Poly(L-lactic acid) & & Filtration \\
\hline Kim 2010 & S/M hybrid & Poly(lactic-co-glycolic acid) & $\begin{array}{l}2800(\mathrm{~S}) \\
530(\mathrm{M})\end{array}$ & Biomedical (scaffolding) \\
\hline $\begin{array}{l}\text { Scholten } 2011 \\
\text { [7] }\end{array}$ & Solution & Polyurethane & Low 1000s & Air filtration (removal of volatile organic compounds \\
\hline
\end{tabular}




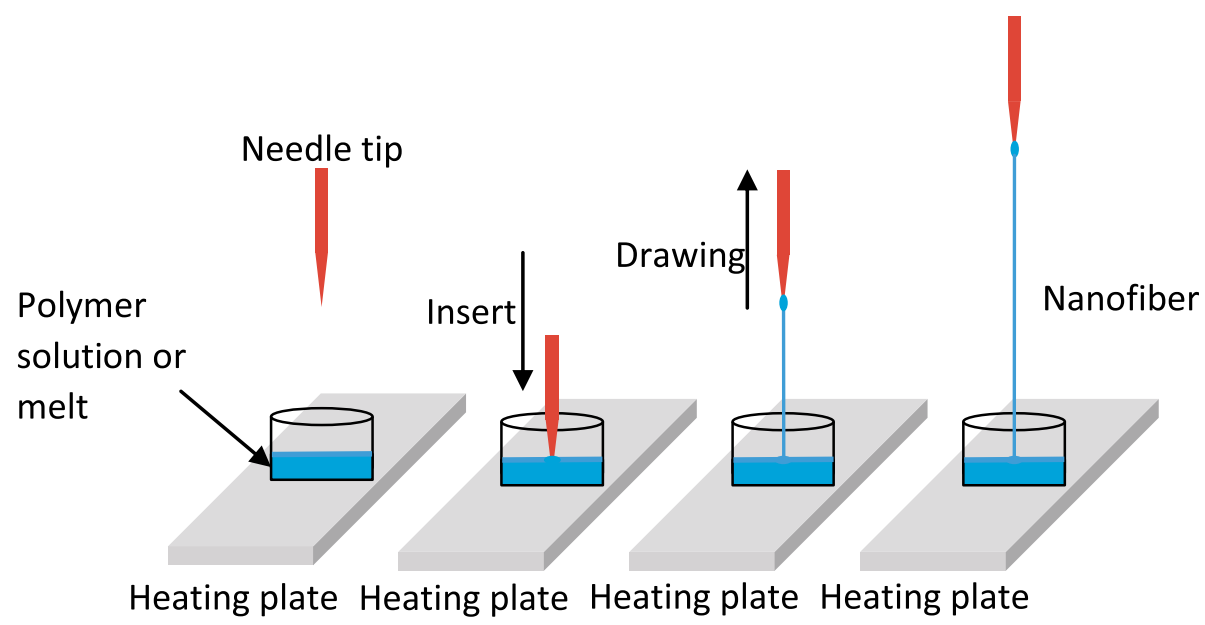

Fig. 4 Basic setup of the fiber-drawing production method

over into the mature fiber [57]. Electrospinning is compatible with many different polymers and multiple applications (Table 1).

Another common method for nanofiber production is fiber drawing. Here, a solid tip is placed in contact with a liquid polymer and then drawn away, leaving behind a string of polymer liquid that solidifies into a fiber (Fig. 4). Like electrospinning, this method is compatible with polymer melts [58] and polymer solutions [46]. One of the main advantages of this method is that it allows the evaluation of single fibers [59]. Drawing typically produces narrower fibers than electrospinning, with diameters of tens of nanometers (Table 2).

The island-in-the-sea method (Fig. 5) is a subtype of conventional melt spinning, but two different polymers are involved. One of them (the sea polymer) is spun into a thick fiber within which multiple thinner fibers of the other (the island polymer) are suspended. Following primary extrusion, the sea polymer is removed to leave the nanoscale island-polymer fibers behind [60]. This method has been used to create polyamide 6/polyethylene terephthalate nanofibers with a consistent diameter of $39 \mathrm{~nm}$ [61].
Melt-blown fibers are produced by extruding a polymer melt through small nozzles surrounded by high-speed flowing gas, typically resulting in microfibers approximately $2 \mu \mathrm{m}$ in diameter (Fig. 6). However, individual sub-microfibers/nanofibers with diameters of $\sim 100 \mathrm{~nm}$ have been produced using an ideal setup comprising an annual air die, Finaplas polypropylene (PP) with a melt flow rate (MFR) of 35 as the polymer, a polymer temperature of $290^{\circ} \mathrm{C}$, a gas temperature of $400^{\circ} \mathrm{C}$ and a feed rate of $4.11 \cdot 10^{-6} \mathrm{~kg} / \mathrm{s}$ [62]. Like electrospinning, which can be scaled up by multiplying the number of jets, melt-blowing can be scaled up by multiplying the number of nozzles to reduce costs [63]. However, unlike electrospinning, which can produce aligned fibers, melt-blown fibers are deposited randomly into non-woven sheets. These are particularly suitable for filtration applications, but melt blowing cannot be used for applications that require oriented fiber sheets.

A derivative of the melt-blowing technique is the Laval spinning method, which also uses an airstream to draw the fiber from the nozzle [64]. However, the shape of the longitudinal Laval nozzle accelerates the air, making the process more efficient than conventional melt-blowing.

Table 2 Overview of non-electrospinning methods, typical fiber diameters and applications

\begin{tabular}{|c|c|c|c|c|}
\hline Publication & Production method & Polymer & Fiber diameter [nm] & Application \\
\hline Xing 2008 [58] & Drawing & Poly(trimethylene terephthalate) & 60 & Optical fibers \\
\hline Ma 2016 [46] & Drawing & Polyethylene & 40 & \\
\hline Nakata 2007 [61] & Island in the sea & Polyamide $6 /$ poly(ethylene terephthalate) & 39 & \\
\hline Uppal 2012 [63] & Melt-blown & & 290 & Filtration \\
\hline Luo [97] & Template synthesis & Silver/cross-linked poly(vinyl alcohol) & Sub-micro & Various \\
\hline Wang 2010 [98] & Template synthesis & Carbon & 20 & Various \\
\hline Rolandi 2014 [99] & Self-assembly & Chitin & various & Various \\
\hline Xu 2017 [100] & Self-assembly & Polypeptide & & Drug delivery \\
\hline Hammami 2014 [74] & Centrifugal & Polyamide 6 & $200-800$ & Various \\
\hline
\end{tabular}




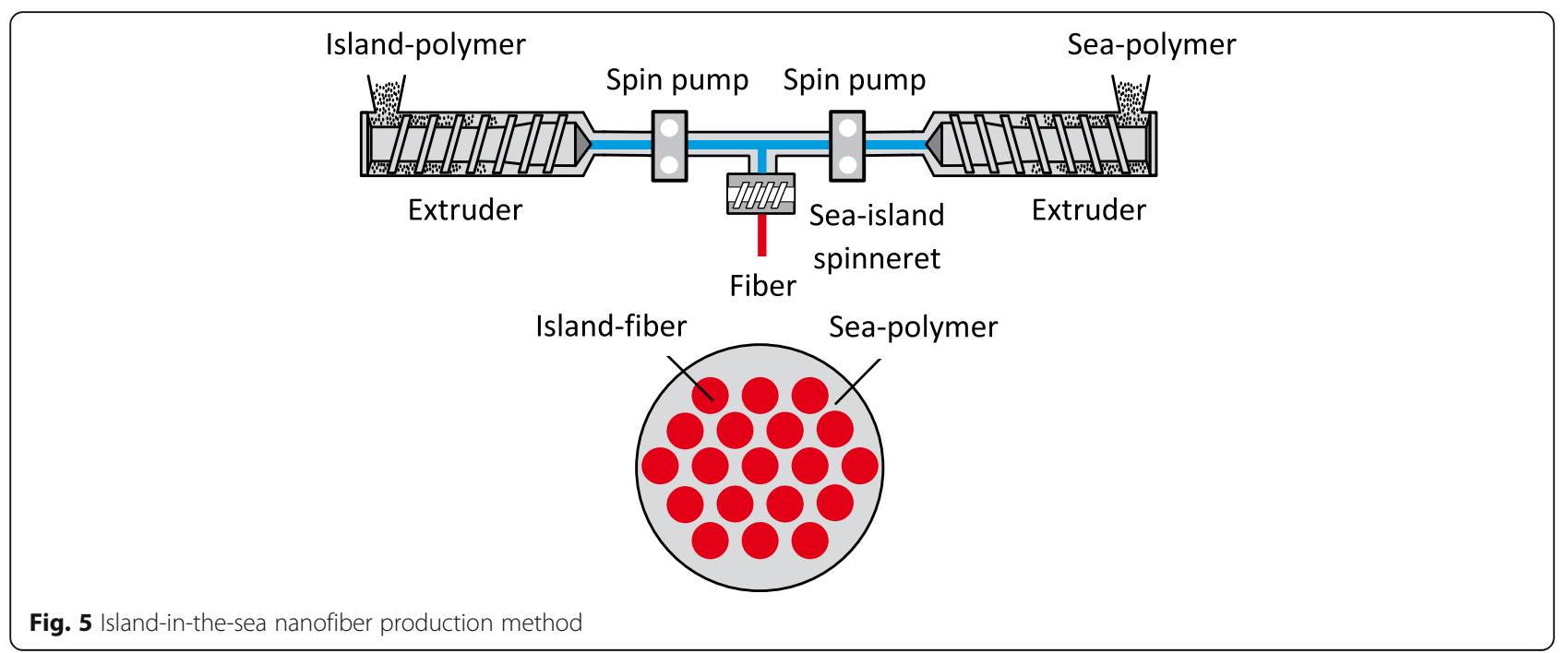

Furthermore, a cold airstream is used rather than the hot airstream of the conventional method (Fig. 7). The laminar airstream enters the nozzle from the back. The nozzle narrows just beyond the entrance channel for the polymer, which accelerates the air stream and the fiber to supersonic velocity. The main advantage compared to conventional melt-blowing is that the nozzle diameter can be much larger, allowing spinning with a high mass throughput per nozzle [64]. The proprietary Nanoval process is similar to the Laval method but it produces a multitude of smaller-diameter fibers that erupt from the original drawn string when the steadily increasing laminar airflow reaches a particular threshold [65].

Electroblowing is essentially a hybrid of electrospinning and blowing $[64,66]$. The voltage at the spinning nozzle is sufficient to allow Taylor cone formation. The fiber is then caught by a low-velocity airstream that carries it away from the spinneret in a manner similar to conventional melt blowing. However, in contrast to conventional melt blowing, electrostatic repulsion is the main force that pulls the fiber from the nozzle and the purpose of the airflow is to reduce interference from the electric field of adjacent nozzles, making the process easier to scale up [64]. Like electrospinning, electroblowing has two variants: solution electroblowing and melt electroblowing, the latter illustrated in Fig. 8. In both cases, the airstream also cools down the liquid fiber to solidify it (melt electroblowing) or to dry it and remove the solvent (solution electroblowing).

Flash spinning is a special form of solution spinning, in which the solvent is a hydrocarbon such as butane or isobutene, which would exist as gas under normal atmospheric pressure at room temperature. The spinning solution is maintained under very high pressure at temperatures of $130-500{ }^{\circ} \mathrm{C}$. When the spinning solution is ejected into an environment with a much lower pressure and temperature, the fiber dries immediately $[67,68]$.

Other, lesser-used production methods include template synthesis and self-assembly. Template synthesis is suitable for the production of both fibers and tubules $[50,69]$. It uses the pores of a host material as a template to control the growth of new materials [57]. For example, polymers can be produced electrochemically by applying a metal layer to a membrane with pores within which the polymers are synthesized [57]. Figure 9 demonstrates the procedure and shows how the fiber diameter and length are controlled by the pore dimensions. [70] Self-assembly is used for the production of nanofibers comprising polypeptides with an intrinsic capacity

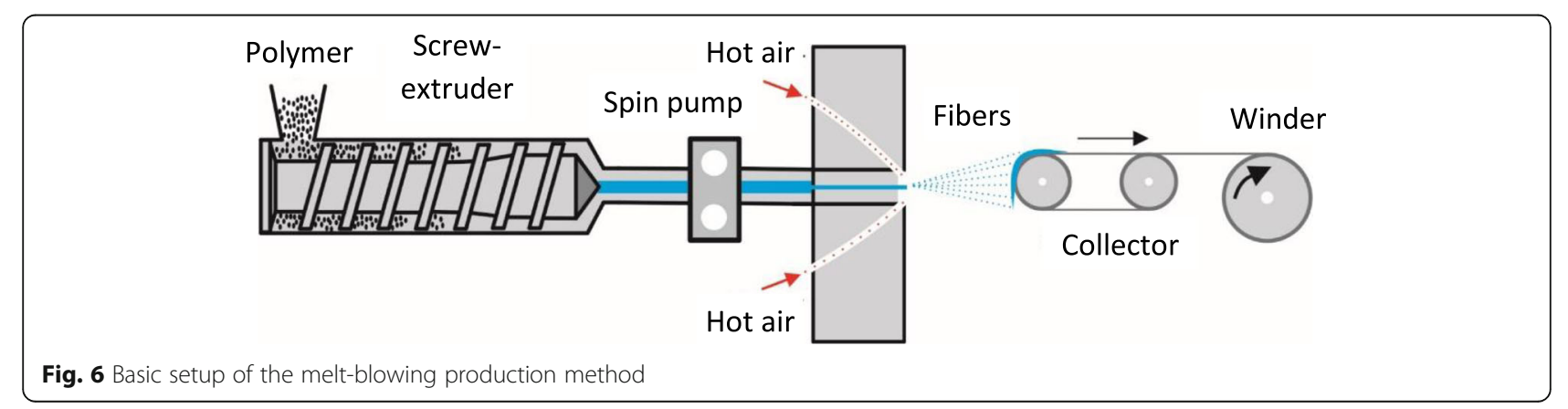




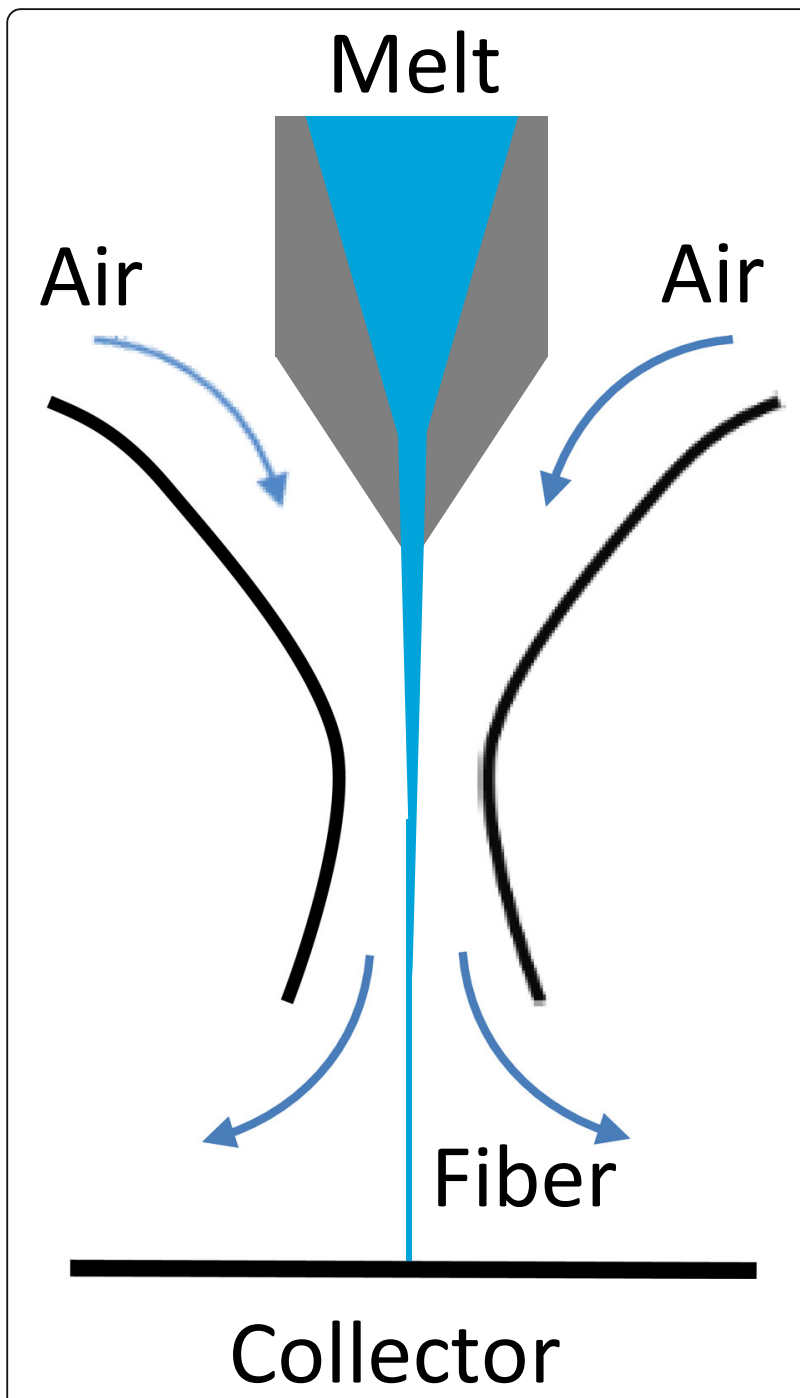

Fig. 7 Airflow through the Laval longitudinal nozzle for self-assembly [71]. The method is based on the spontaneous organization of individual macromolecules into an ordered and stable nanoscale structure [41]. A solution is necessary to create the appropriate environment for the formation of these structures, which have a potential minimum diameter of $3 \mathrm{~nm}$ [72]. Although very small diameters can be achieved, this technology is complex and has a low throughput, making it difficult to scale up and thus unsuitable for industrial applications [41].

Whereas melt blowing and its variations are easy to scale up, these methods cannot produce oriented fibers. In contrast, the other methods can produce oriented fibers but are hampered by their low throughput. Centrifugal spinning can overcome this challenge by mounting the spinneret on a centrifuge with the nozzles facing outward [73, 74]. When the centrifugal force (dependent on the rotor diameter and angular velocity) is sufficient to overcome the drag caused by the viscosity of the polymer solution or melt, a steady polymer jet streams from the nozzle to the collector [73, 74]. The centrifugal spinning method is shown in Fig. 10. A derivative of this method is split-fiber production, where the nozzle is split into several smaller nozzles to produce narrower fibers or flat bands [75]. The throughput is up to 500-fold higher than conventional solution electrospinning [76]. However, the use of solvents and the strong dependence on the elasticity of the polymer solution and the evaporation rate of the solvents make this process difficult to control [77].

\section{Development of a new melt-electrospinning prototype for sub-microfibers and nanofibers} Work leading up to the prototype - state of the art Electrospinning methods allow the production of single or multiple nanofibers (depending on the number of jets)

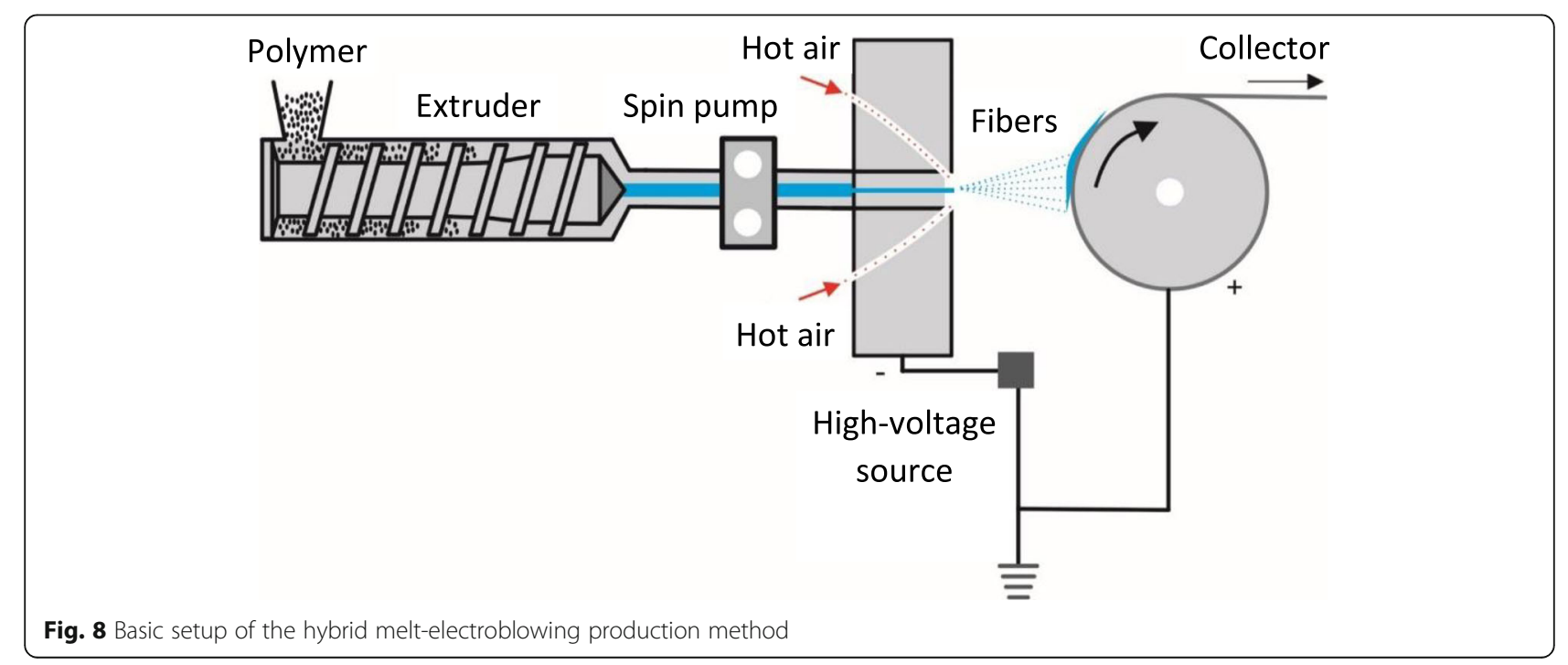




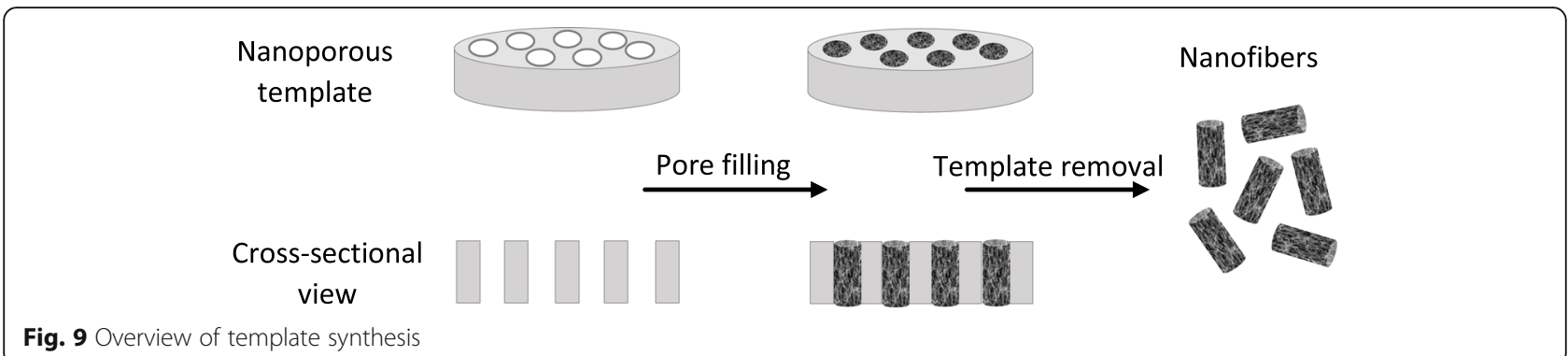

using a simple apparatus with relatively low setup and operating costs, so electrospinning can be an economically competitive production method [78]. The presence of solvent in the solution electrospinning process adds an expensive recovery step to the overall manufacturing process, and the potential carryover of toxic solvents or solvents with undesirable optical activity makes solution electrospinning unsuitable for medical and filtration applications or the production of optical sensors. Although the high temperature, high viscosity and low conductivity of the molten polymer is a challenge that must be addressed during melt electrospinning $[79,80]$, the absence of solvent ensures stable jet formation, allowing the direct deposition of micrometer to sub-micrometer range fibers and the reproduction of three-dimensional structures [81-85]. Melt electrospinning is not compatible with non-thermoplastic materials, including biological polymers such as collagen, but is ideal for sparingly-soluble polymers such as PP and polyethylene. Other commonly used polymers include PCL, PU, PLA, and PLGA. [86-92].

In order to produce nanoscale fibers, the polymer delivery rate during melt electrospinning must be significantly lower than during solution electrospinning, which explains the absence of melt electrospinning as an industrial manufacturing method for nanofibers [4]. However, only $2-10 \%$ of the liquid processed during solution electrospinning is the polymer (the rest is solvent that evaporates) whereas $100 \%$ of the processed liquid solidifies into fibers during melt electrospinning, indicating that the industrial use of this method could be achieved by scaling the process up [4]. Accordingly, recent device innovations, such as multiple-needle and needleless configurations, have demonstrated a roadmap to overcome the low throughput of melt electrospinning, typically in the $\mu \mathrm{g} / \mathrm{h}$ range [4]. Prototypes with umbellate nozzles containing 60 spinnerets can achieve maximum product deposition rates of $\sim 36 \mathrm{~g} / \mathrm{h}[93$,

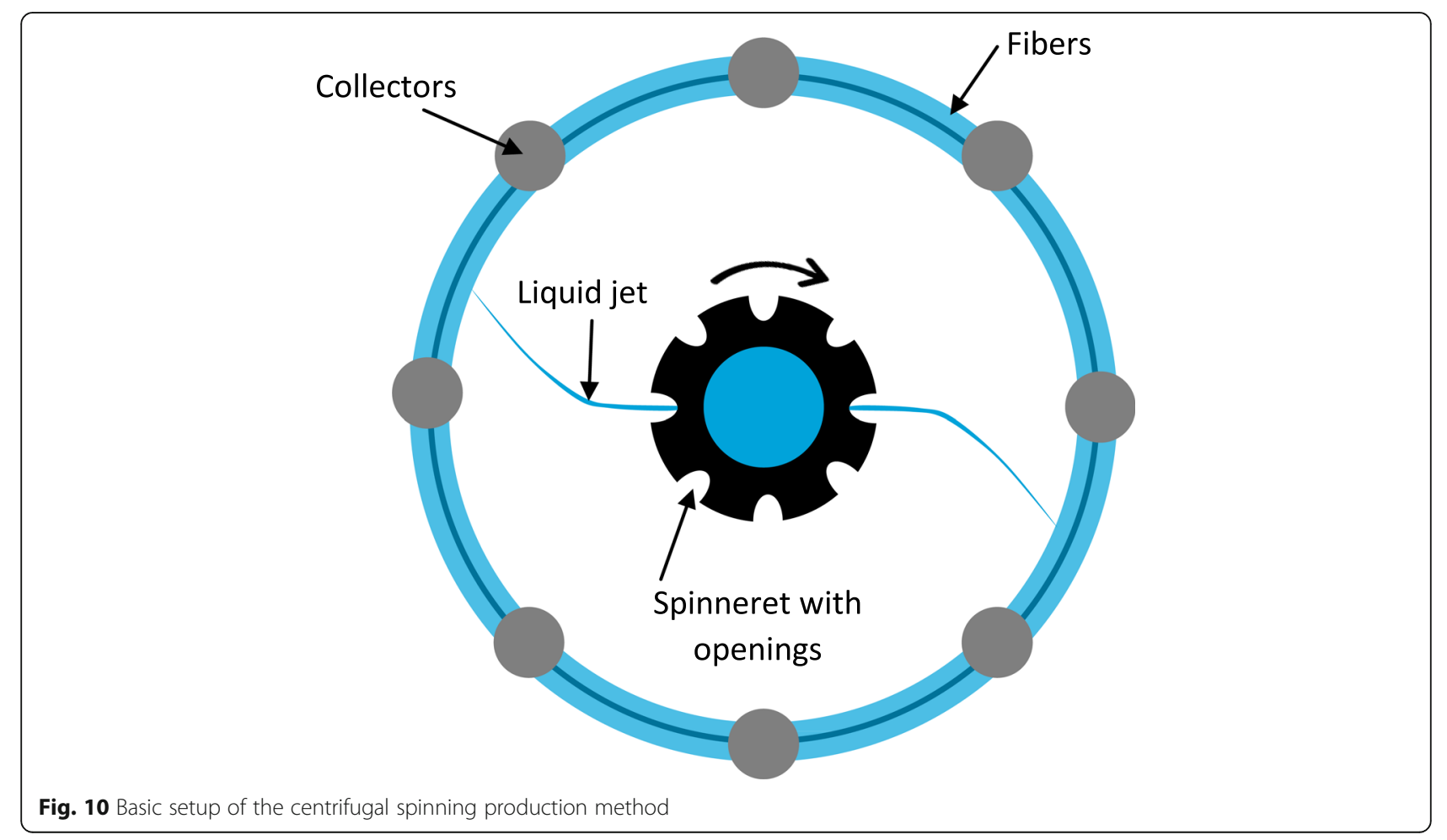




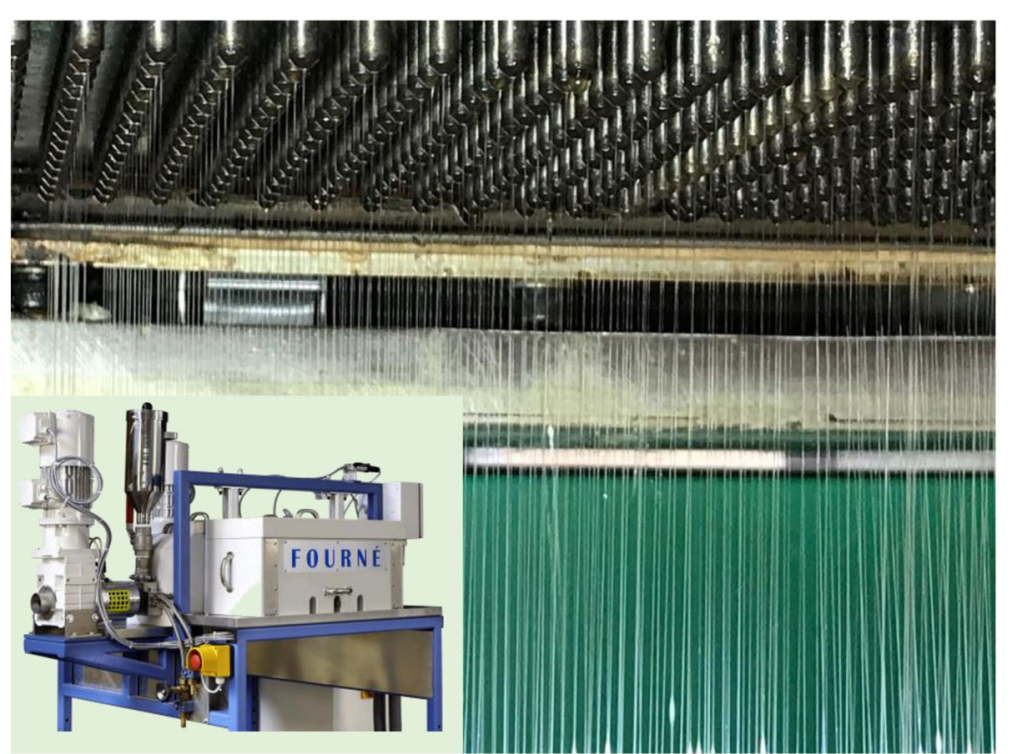

Fig. 11 The 600-nozzle melt-electrospinning prototype developed at the AMIBM

94]. The largest multi-nozzle spinning device described in the literature thus far features 64 nozzles [95].

\section{Prototype for the scaled-up melt electrospinning of sub- microfibers and nanofibers}

The Aachen-Maastricht Institute for Biobased Materials (AMIBM) at Maastricht University has cooperated with Fourné Maschinenbau GmbH (Alfter-Impekoven, Germany) and Pötter-Klima Gesellschaft für Nanoheiztechnik mbH (Georgsmarienhütte, Germany) to develop a functional prototype of a melt-electrospinning device featuring a spinneret with 600 nozzles, which vastly exceeds the capabilities of any state-of-the-art technologies. The nozzle plate of this device is shown in Fig. 11. Each nozzle has a diameter of $0.3 \mathrm{~mm}$ and the nozzles are spaced $8 \mathrm{~mm}$ apart.

One of the major challenges when scaling up a melt-electrospinning device from a smaller number to a larger number of spinnerets is the uniform distribution of the melt to all nozzles. The low-volume flow of the polymer melt during melt electrospinning may lead to incomplete nozzle filling, resulting in sporadic and unpredictable pressure losses within each nozzle. Inside the prototype nozzle, melt flow has been improved by taking this design consideration into account and introducing a three-plate construction and two symmetrically designed polymer inlets. A distributor plate combined with a finely porous sintered plate ensures the optimum melt distribution and a uniform pressure build-up over the entire nozzle cross-section. A relatively high specific contact load at the sealing line as well as the use of aluminum flanges guarantees the sealing of the nozzle plates. The constant supply of polymer melt is ensured by a speed-adjustable single-screw extruder and spinning pump.

Another challenge addressed by the new prototype is the tendency for solidified polymer to block the capillaries. The integration of heating elements around the spinneret

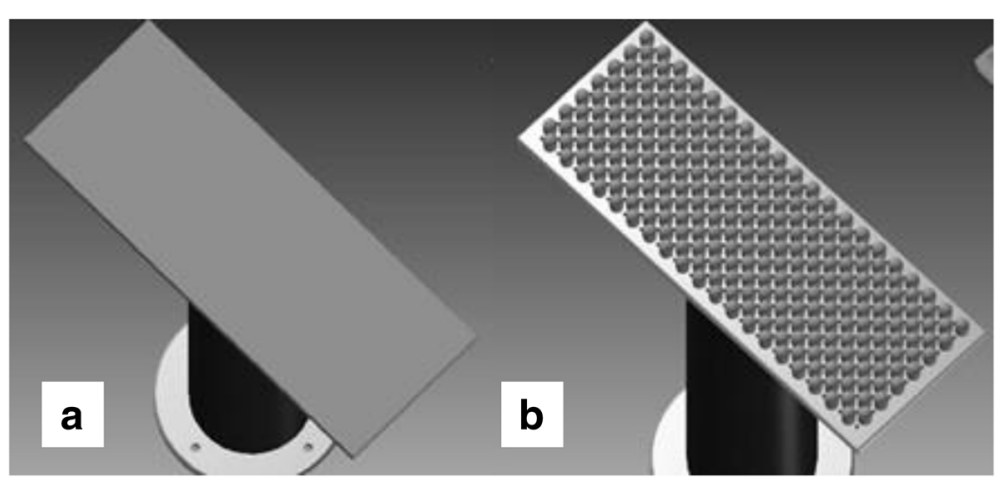

Fig. 12 Comparison of (a) a conventional collector and (b) the novel collector designed for the 600-nozzle AMIBM melt-electrospinning prototype 


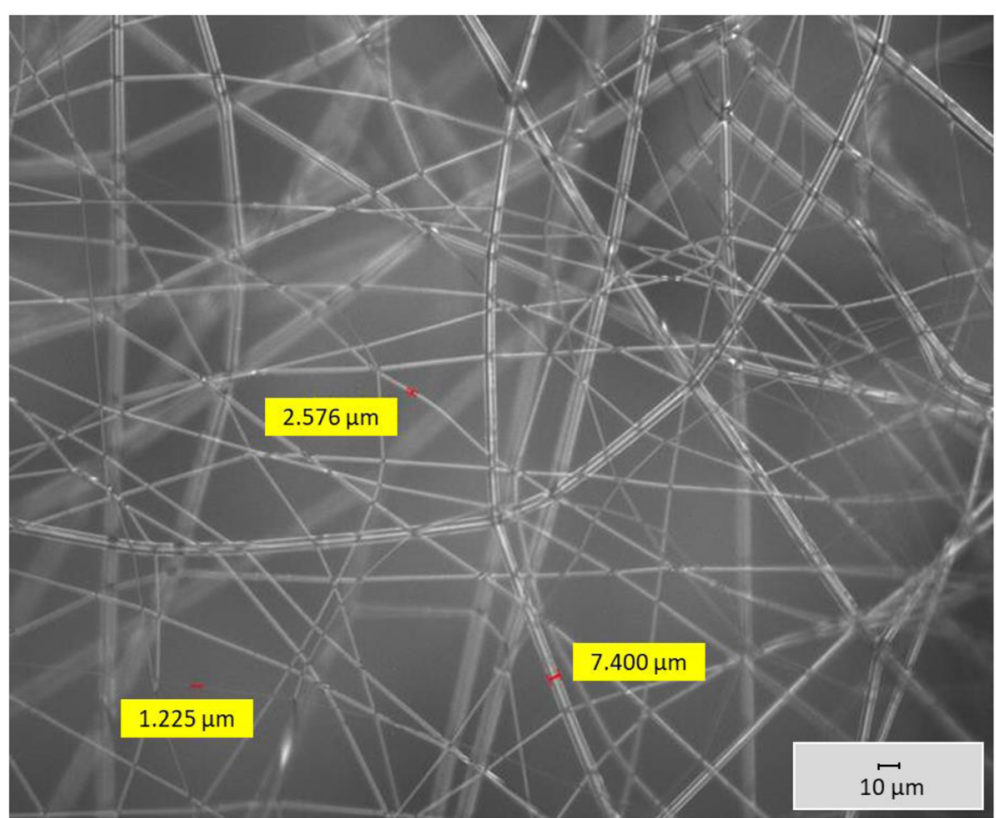

Fig. 13 PLA microfibers containing 6\% (w/w) sodium stearate produced using the 600-nozzle AMIBM melt-electrospinning prototype

achieves a uniform polymer melt flow from the nozzles to prevent this common problem during fiber production. A collector with an uneven surface is used instead of a conventional plate collector to facilitate the optimal spreading of the collected fibers (Fig. 12). With the nozzle/collector pairing installed in the prototype, nonwovens can be produced continuously over a width of $340 \mathrm{~mm}$. The collector is connected to an Eltex KNH65 source supplying a positive high voltage $(1-60 \mathrm{kV})$ while simultaneously grounding the spinneret.

Initially, the device was used to produce PP fibers containing conductive additives, and the finest fiber had a diameter of $6.64 \mu \mathrm{m}$. This was produced using high-flow PP HL508FB (Borealis AG, Vienna, Austria) containing 2\% $(w / w)$ sodium stearate (Alfa Aesar, Karlsruhe, Germany). The distance between the collector and the nozzle plate was $11 \mathrm{~cm}$, a positive voltage of $60 \mathrm{kV}$ was applied to the collector, the nozzle was heated to $210^{\circ} \mathrm{C}$ and the polymer flow rate was defined by a spinning pump speed of $16 \mathrm{rpm}$ [96]. Having verified the function of the device, we then attempted the production of biobased fibers using Ingeo Biopolymer 6201D, a commercial spinning-grade PLA (NatureWorks LLC, Minnetonka, Minnesota, USA) containing $6 \%(\mathrm{w} / \mathrm{w})$ sodium stearate. We maintained the distance between the collector and nozzle plate at $11 \mathrm{~cm}$ but reduced the nozzle temperature to $190^{\circ} \mathrm{C}$ and the spinning pump speed to $2 \mathrm{rpm}$, yielding fibers ranging from 1.000 to $7.000 \mu \mathrm{m}$ in diameter (Fig. 13).

\section{Outlook}

Several methods can be used to produce nanofibers and sub-microfibers, but melt electrospinning is among the most promising technologies in terms of fiber structure and the breadth of downstream applications due to the absence of solvents in the manufacturing process. The major drawback of melt electrospinning is its low throughput, resulting in the adoption of solution electrospinning as the principal industrial process technology. Although some attempts have been made to scale up the electrospinning method, an industrial process has yet to be established. At the AMIBM, we have developed a promising, scaled-up melt-electrospinning prototype that bridges the gap between laboratory-scale and pilot-scale manufacturing. Thus far, we have produced PLA fibers $\sim 1 \mu \mathrm{m}$ in diameter, but this was achieved without comprehensive optimization of the apparatus, the process parameters or the polymer substrate and additives. There are many opportunities to improve the performance of the device by adding new features such as a controllable climate chamber around the spinneret to improve jet stretching before the collector, delaying the solidification of the melt and thus producing thinner fibers with uniform diameters. In the future, individually controlled collector tips in a multi-nozzle structure with the writing ability of melt electrospinning could lead to the development of truly innovative microfiber and nanofiber products.

\section{Abbreviations}

AMIBM: Aachen-Maastricht Institute for Biobased Materials; DIN: Deutsches Institut für Normung; MFR: Melt flow rate; PCL: Polycaprolactone; PLA: Polylactic acid; PLGA: Poly(lactic-co-glycolic) acid; PP: Polypropylene; PU: Polyurethane 


\section{Acknowledgements}

The authors acknowledge the support of Fourné Maschinenbau GmbH (Alfter-Impekoven, Germany), Pötter-Klima Gesellschaft für Nanoheiztechnik mbH (Georgsmarienhütte, Germany), Schnick Systemtechnik GmbH \& Co KG (Heiligenhaus,Germany), and the Chair of Polymer Materials (LSP) of Erlangen-Nürnberg University (Germany). We also thank our Bachelor's students Nina Goebel and Jaqueline Ellerkmann for their support with the literature research and preparation of figures, and our hands-on technician Henri Becker.

\section{Funding}

Not applicable

\section{Availability of data and materials}

The datasets used and/or analyzed during the current study are available from the corresponding author on reasonable request.

\section{Authors' contributions}

KK performed the spinning trials, material development, and wrote the manuscript. KB supported the literature research, the spinning trials and analyzed the fibers. FL and GS helped with the plant design. All authors read and approved the final manuscript.

\section{Ethics approval and consent to participate}

Not applicable

\section{Consent for publication}

Not applicable

\section{Competing interests}

The authors declare that they have no competing interests.

\section{Publisher's Note}

Springer Nature remains neutral with regard to jurisdictional claims in published maps and institutional affiliations.

\section{Received: 20 February 2019 Accepted: 20 March 2019}

Published online: 29 March 2019

\section{References}

1. Back S. The role of nanotechnology in sustainable textiles. BLACKBURN, R. S.: Sustainable Textiles. Woodhead Publishing. , 2009. 978-1-84569-453-1 p. 302-304.

2. DIN CEN ISO/TS 80004-1: Nanotechnologien - Fachwörterverzeichnis - Teil 1: Kernbegriffe (ISO/TS 80004-1:2015); Deutsche Fassung CEN ISO/TS 80004-1:2015

3. DIN CEN ISO/TS 80004-2: Nanotechnologien - Fachwörterverzeichnis - Teil 2: Nanoobjekte (ISO/TS 80004-2:2015); Deutsche Fassung CEN ISO/TS 80004-2:2017.

4. Brown T, Dalton P, Hutmacher DW, et al. Melt electrospinning today: an opportune time for an emerging polymer process. Elsevier. 2016;56:116-66.

5. Bhat GS. Advances in polymeric nanofiber manufacturing technologies. J Nanomater Mol Nanotechnol. 2016;5:2324.

6. Sundarrajan $\mathrm{S}$, Tan $\mathrm{KL}$, Lim SH, et al. Electrospun nanofibers for air filtration applications. Procedia Eng. 2014;75:159-63.

7. Scholten E, Bromberg L, Rutledge GC, et al. Electrospun polyurethane fibers for absorption of volatile organic compounds from air. ACS Appl Mater Interfaces. 2011:3:3902-9.

8. Sarbatly R, Krishnaiah D, Kamin Z. A review of polymer nanofibres by electrospinning and their application in oil-water separation for cleaning up marine oil spills. Mar Pollut Bull. 2016;106:8-16.

9. Song M, Park S, Alamgir F, et al. Nanostructured electrodes for lithium-ion and lithium-air batteries: the latest developments, challenges, and perspectives. Mater Sci Eng R Reports. 2011;72:203-52.

10. Wang J, Li Y, Sun X. Challenges and opportunities of nanostructured materials for aprotic rechargeable lithium-air batteries. Nano Energy. 2013;2: 443-67.

11. Zhang G, Zheng J, Liang R, et al. a-MnO2/carbon nanotube/carbon nanofiber composite catalytic air electrodes for rechargeable lithium-air batteries. jes.ecsdl.org 2011; 158: A822.
12. Truong TT, Liu Y, Ren Y, et al. Morphological and crystalline evolution of nanostructured $\mathrm{MnO}_{2}$ and its application in lithium-air batteries. ACS Nano. 2012;6:8067-77

13. Wang $X$, Drew $\mathrm{C}$, Lee $\mathrm{S}-\mathrm{H}$, et al. Electrospun Nanofibrous membranes for highly sensitive optical sensors. Nano Lett. 2002;2:1273-5.

14. Bhardwaj N, Kundu SC. Electrospinning: a fascinating fiber fabrication technique. Biotechnol Adv. 2010;28:325-47.

15. Ma B, Xie J, Jiang J, et al. Rational design of nanofiber scaffolds for orthopedic tissue repair and regeneration. Nanomedicine (Lond). 2013;8: 1459-81.

16. Shin $\mathrm{S}-\mathrm{H}$, Purevdorj $\mathrm{O}$, Castano $\mathrm{O}$, et al. A short review: recent advances in electrospinning for bone tissue regeneration. J Tissue Eng. 2012;3:1-9.

17. McClellan P, Landis WJ. Recent applications of coaxial and emulsion electrospinning methods in the field of tissue engineering. Biores Open Access. 2016:5:212-27.

18. Cui W, Zhou Y, Chang J. Electrospun nanofibrous materials for tissue engineering and drug delivery. Sci Technol Adv Mater. 2010;11:014108.

19. Sill T, von Recum HA. Electrospinning: applications in drug delivery and tissue engineering. Biomaterials. 2008;29(13):1989-2006.

20. Yoo H, Kim TG, Park TG. Surface-functionalized electrospun nanofibers for tissue engineering and drug delivery. Adv Drug Deliv Rev. 2009; 61(12):1033-42.

21. Khajavi R, Abbasipour M, Bahador A. Electrospun biodegradable nanofibers scaffolds for bone tissue engineering. J Appl Polym Sci. 2016;133:42883.

22. Venugopal J, Low S, Choon AT, et al. Interaction of cells and nanofiber scaffolds in tissue engineering. J Biomed Mater Res Part B Appl Biomater. 2008;84(1):34-48.

23. Vasita R, Katti DS. Nanofibers and their applications in tissue engineering. Int J Nanomedicine. 2006;1:15-30.

24. Chew SY, Wen Y, Dzenis $Y$, et al. The role of electrospinning in the emerging field of nanomedicine. Curr Pharm Des. 2006;12:4751-70.

25. Chakraborty S, Liao I-C, Adler A, et al. Electrohydrodynamics: a facile technique to fabricate drug delivery systems. Adv Drug Deliv Rev. 2009;61: 1043-54.

26. Goyal R, Macri LK, Kaplan HM, et al. Nanoparticles and nanofibers for topical drug delivery. J Control Release. 2016:240:77-92.

27. Zamani M, Prabhakaran MP, Ramakrishna S. Advances in drug delivery via electrospun and electrosprayed nanomaterials. Int J Nanomedicine. 2013;8: 2997-3017.

28. Weng L, Xie J. Smart electrospun nanofibers for controlled drug release: recent advances and new perspectives. Curr Pharm Des. 2015;21:1944-59.

29. Chou S-F, Carson D, Woodrow KA. Current strategies for sustaining drug release from electrospun nanofibers. J Control Release. 2015;220:584-91.

30. Zeng J, Xu X, Chen X, et al. Biodegradable electrospun fibers for drug delivery. Elsevier. 2003;92:227-31.

31. Katti DS, Robinson KW, Ko FK, et al. Bioresorbable nanofiber-based systems for wound healing and drug delivery: optimization of fabrication parameters. J Biomed Mater Res. 2004;70(2):286-96.

32. Hu X, Liu S, Zhou G, et al. Electrospinning of polymeric nanofibers for drug delivery applications. Elsevier. 2014;185:12-21.

33. Kenawy E, Abdel-Hay, El-Newehy $\mathrm{MH}$, et al. Processing of polyme nanofibers through electrospinning as drug delivery systems. Elsevier. 2009; 113:296-302.

34. Shen $X, Y u$ D, Zhu $L$, et al. Electrospun diclofenac sodium loaded Eudragit ${ }^{\circledR}$ L 100-55 nanofibers for colon-targeted drug delivery. Int J Pharm. 2011;408: 200-7.

35. Pillay $V$, Dott $C$, Choonara $Y$, et al. A review of the effect of processing variables on the fabrication of electrospun nanofibers for drug delivery applications. hindawi.com 2013; 2013: 22.

36. Naves LB, Dhand C, Venugopal JR, et al. Nanotechnology for the treatment of melanoma skin cancer. Prog Biomater. 2017;6:13-26.

37. Chen Z, Chen Z, Zhang A, et al. Electrospun nanofibers for cancer diagnosis and therapy. Biomater Sci. 2016;4:922-32.

38. Kim Y-J, Ebara M, Aoyagi T. A smart hyperthermia nanofiber with switchable drug release for inducing Cancer apoptosis. Adv Funct Mater. 2013:23:5753-61.

39. Shi D, Bedford NM, Cho H-S. Engineered multifunctional Nanocarriers for Cancer diagnosis and therapeutics. Small. 2011;7:2549-67.

40. Zhang N, Deng Y, Tai Q, et al. Electrospun TiO2 nanofiber-based cell capture assay for detecting circulating tumor cells from colorectal and gastric Cancer patients. Adv Mater. 2012;24:2756-60. 
41. Kwnry, Lim CT: Nanofiber technology: current status and emerging developments. In: Topical Volume on Advanced Polymeric Materials Bd. 2017; 70: 1-17.

42. Blim A, Jarecki L, Blonski S. Modeling of pneumatic melt drawing of polypropylene super-thin fibers in the Laval nozzle. Bull Pol Ac. 2014;62:42-54.

43. NANOVAL GMBH \& CO KG: Nanoval - Process. https://www.nanoval.de/ index.php. Accessed 17 Oct 2018.

44. Badrossamay MR, Mcllwee HA, Goss JA, Parker KK. Nanofiber assembly by rotary jet-spinning. Nano Lett. 2010;10(6):2257-6.

45. Medeiros ES, Glenn GM, Klamcynski AP, Orts WJ, Mattoso LH. Solution blow spinning: a new method to produce micro- and nanofibers from polymer solutions. J Appl Polym Sci. 2009;113(4):2322-30.

46. Ma J, Zhang Q, Zhang Y, et al. A rapid and simple method to draw polyethylene nanofibers with enhanced thermal conductivity. Appl Phys Lett. 2016;109:033101.

47. Masuda M, Funakoshi J. Island-in-sea fiber, combined filament yarn and textile product. EP2821533A1 2012.

48. Biswas A, Bayer IS, Biris AS, Wang T, Dervishi E, et al. Advances in top-down and bottom-up surface nanofabrication: techniques, applications \& future prospects. Adv Colloid Interf Sci. 2012;170(1):2-27.

49. Barnes CP, Sell SA, Boland ED, Simpson DG, Bowlin GL. Nanofiber technology: designing the next generation of tissue engineering scaffolds. Adv Drug Deliv Rev. 2007;59(14):1413-33.

50. Martin CR. Template synthesis of electronically conductive polymer nanostructures. Acc Chem Res. 1995;28:61-8.

51. Suvorov VG, Zubarev NM. Formation of the Taylor cone on the surface of liquid metal in the presence of an electric field. J Phys D Appl Phys. 2004;37: 289-97.

52. Suvorov VG, Litvinov EA. Dynamic Taylor cone formation on liquid metal surface: numerical modelling. J Phys D Appl Phys. 2000;33:1245-51.

53. Yarin $\mathrm{AL}$, Koombhongse $\mathrm{S}$, Reneker $\mathrm{DH}$. Taylor cone and jetting from liquid droplets in electrospinning of nanofibers. J Appl Phys. 2001;90:4836-46.

54. Li D, Xia Y. Electrospinning of nanofibers: reinventing the wheel? Adv Mater. 2004;16:1151-70.

55. Ramakrishna S, Fujihara K, Teo W-E, et al. Electrospun nanofibers: solving global issues. Mater Today. 2006:9:40-50.

56. Ahn YC, Park SK, Kim GT, Hwang XY, Lee CG et al. Development of high efficiency nanofilters made of nanofibers. Nano Korea 2004 Symposium on NT Challenge 2006; 6(6): 1030-1035

57. Hutmacher DW, Dalton PD. Melt electrospinning. Chem - An Asian J. 2011;6: 44-56.

58. Xing $X$, Wang $Y$, Li B. Nanofibers drawing and nanodevices assembly in poly(trimethylene terephthalate). Opt Express. 2008;16:10815.

59. Bajakova J, Chaloupek J, et al. "Drawing"- The production of individual nanofibers by experimental method. Nanocon. 2011.

60. Ndaro MS, Jin $X$, Chen T, et al. Splitting of islands-in-the-sea fibers ( PA6/COPET ) during hydroentangling of nonwovens. J Eng Fiber Fabr. 2007:2:1-9.

61. Nakata K, Fujii K, Ohkoshi Y, et al. Poly(ethylene terephthalate) nanofibers made by Sea-Island-type conjugated melt spinning and laser-heated flow drawing. Macromol Rapid Commun. 2007;28:792-5.

62. Shambaugh RL. A macroscopic view of the melt-blowing process for producing microfibers. Ind Eng Chem Res. 1988;27:2363-72.

63. Uppal R, Bhat G, Eash C, et al. Meltblown nanofiber media for enhanced quality factor. Fibers Polym. 2013;14:660-8.

64. Yoon K, Hsiao BS, Chu B. Functional nanofibers for environmental applications. J Mater Chem. 2008;18:5326-34.

65. Gerkin L. Nanoval process for spunbonds detailed. Int Fiber J. 2005;20:52-6.

66. Kim YM, Ahn KR, Sung YB, et al. Manufacturing device and the method of preparing for the nanofibers viaelectro-blown spining process. US 7,618,579 B2, USPTO, 2011.

67. Palmer L. Flash spinning. US3565979A, USPTO, 1968.

68. Nayak R, Padhye R, Kyratzis IL, et al. Recent advances in nanofibre fabrication techniques. Text Res J. 2012;82:129-47.

69. Martin CR. Nanomaterials: a membrane-based synthetic approach. Science. 1994;266:1961-6.

70. Karatas A, Algan AH. KARATAŞ.Template synthesis of tubular nanostructures for loading biologically active molecules. Curr Top Med Chem. 2016;17.

71. Malkar NB, Lauer-Fields $J L$, Juska D, et al. Characterization of peptide -Amphiphiles possessing cellular activation sequences. Biomacromolecules. 2003:4:518-28.
72. Hassanzadeh P, Kharaziha M, Nikkhah M, Shin SR, Jin J, et al. Chitin nanofiber micropatterned flexible substrates for tissue engineering. J Mater Chem. 2013;1(34):4217-24.

73. Zhang $X$, Lu Y. Centrifugal spinning: an alternative approach to fabricate nanofibers at high speed and low cost. Polym Rev. 2014;54:677-701.

74. Hammami MA, Krifa M, Harzallah O. Centrifugal force spinning of PA6 nanofibers - processability and morphology of solution-spun fibers. J Text Inst. 2014;105:637-47.

75. Peno E, Lipton R, Kay S. Split fiber producing devices and methods for the production of microfibers and nanofibers. US8778240B2, USPTO, 2012.

76. Ren L, Ozisik R, Kotha SP. Rapid and efficient fabrication of multilevel structured silica micro-/nanofibers by centrifugal jet spinning. J Colloid Interface Sci. 2014:425:136-42.

77. Ren L, Ozisik R, Kotha SP, Underhill P. Highly efficient fabrication of polymer nanofiber assembly by centrifugal jet spinning: process and characterization. Macromolecules. 2015;48(8):2593-602.

78. Garg K, Bowlin GL. Electrospinning jets and nanofibrous structures. Biomicrofluidics. 2011;5:13403.

79. Nayak R. Polypropylene nanofibers: melt electrospinning versus Meltblowing. Engineering Material: Springer International Publishing; 2017.

80. Bubakir M, Barhoum A, Li H, Yang W. Handbook of nanofibers (1st edtn), springer nature; 2017.

81. Willerth SM. Electrospun materials for tissue engineering and biomedical applications: research. Design and Commercialization: Woodhead Publishing; 2017.

82. Wunner FM, Wille ML, Nanoon TG, Bas O, Dalton PD, et al. Melt electrospinning writing of highly ordered large volume scaffold architectures. Adv Mater. 2018;30:1706570.

83. Wunner FM, Maartens J, Bas O, Gottschalk K, De-Juan-Pardo E, et al. Electrospinning writing with molten poly ( $\varepsilon$-caprolactone) from different directions - examining the effects of gravity. Material Letters. 2018;216: $114-8$.

84. Eichholz KF, Hoey DA. Mediating human stem cell behaviour via defined fibrous architectures by melt electrospinning writing. Acta Biomater. 2018; 75:140-51.

85. Dayan CB, Afghah F, Okan BS, Yıldız M, Menceloglu Y, et al. Modeling 3D melt electrospinning writing by response surface methodology. Mater Des. 2018:148:87-95.

86. Zhao F, Liu Y, Yuan H, et al. Orthogonal design study on factors affecting the degradation of polylactic acid fibers of melt electrospinning. J Appl Polym Sci. 2012;125:2652-8.

87. Li X, Liu H, Liu J, et al. Preparation and experimental parameters analysis of laser melt electrospun poly(L-lactide) fibers via orthogonal design. Polym Eng Sci. 2012;52:1964-7.

88. Larrondo L, St. John Manley R. Electrostatic fiber spinning from polymer melts. I. Experimental observations on fiber formation and properties. J Polym Sci Polym Phys Ed 1981; 19: 909-920.

89. Dalton PD, Lleixà Calvet J, Mourran A, et al. Melt electrospinning of poly(ethylene glycol-block-ع-caprolactone). Biotechnol J. 2006;1:998-1006.

90. Yoon Y II, Park KE, Lee SJ, et al. Fabrication of microfibrous and nano -/microfibrous scaffolds: melt and hybrid electrospinning and surface modification of poly(L-lactic acid) with plasticizer. Biomed Res Int 2013; 2013: 309048

91. Zhou H, Green TB, Joo YL. The thermal effects on electrospinning of polylactic acid melts. Polymer (Guildf). 2006;47:7497-505.

92. Brown TD, Dalton PD, Hutmacher DW. Direct writing by way of melt electrospinning. Adv Mater. 2011;23:5651-7.

93. Li $H$, Chen $H$, Zhong $X$, et al. Interjet distance in needleless melt differential electrospinning with umbellate nozzles. J Appl Polym Sci. 2014;131:40515.

94. Liu Y, Zhao F, Zhang C, Zhang J, Yang W. Solvent-free preparation of poly(lactic acid) fibers by melt electrospinning using an umbrella-like spray head and alleviation of the problematic thermal degradation. J Serb Chem Soc. 2012;77:1071-82.

95. Hacker CJP, Seide G, Gries T, Thomas H, Moeller M. (2009) Electrospinning of polymer melt: steps toward an upscaled multi-jet process. In:proc Int confer latest advances in high tech textiles and textile-based materials: 71-76.

96. Koenig K, Daenicke J, Langensiepen F, Seide G, Schubert DW. From lab to pilot scale: melt electrospun nanofibers of polypropylene with conductive additives. J Nanomater Mol Nanotechnol. 2019;8:1.

97. Luo L-B, Yu S-H, Qian H-S, et al. Large-scale synthesis of flexible gold/crosslinked-PVA sub-microcables and cross-linked-PVA tubes/fibers by using 
templating approaches based on silver/cross-linked-PVA sub-microcables. Chemistry. 2006;12:3320-4.

98. Wang $\mathrm{Y}$, Zheng $\mathrm{M}, \mathrm{Lu} \mathrm{H}$, et al. Template synthesis of carbon nanofibers containing linear mesocage arrays. Nanoscale Res Lett. 2010;5:913-6.

99. Rolandi M, Rolandi R. Self-assembled chitin nanofibers and applications. Adv Colloid Interf Sci. 2014;207:216-22.

100. Xu D, Samways DSK, Dong H. Fabrication of self-assembling nanofibers with optimal cell uptake and therapeutic delivery efficacy. Bioact Mater. 2017;2: 260-8.

Ready to submit your research? Choose BMC and benefit from:

- fast, convenient online submission

- thorough peer review by experienced researchers in your field

- rapid publication on acceptance

- support for research data, including large and complex data types

- gold Open Access which fosters wider collaboration and increased citations

- maximum visibility for your research: over $100 \mathrm{M}$ website views per year

At BMC, research is always in progress.

Learn more biomedcentral.com/submissions 